\title{
Muerte materna incidental en un accidente por relámpago: Reporte de caso
}

\author{
Román Jiménez-López* \\ Indalecio Gustavo Martínez-Velasco** \\ José Antonio Rosas-Díaz*** \\ Neylin Veliz-Pradel**** \\ Andrés Cruz-Rivera*****
}

\begin{abstract}
*Médico especialista en Medicina del Enfermo en Estado Crítico. Subdirector Médico. Hospital de la Madre y el Niño Indígena Guerrerense. Secretaría de Salud de Guerrero. Guerrero. México.

**Médico Ginecobstetra-Perinatólogo. Hospital Integral de Tecomatlán. Secretaria de Salud de Puebla. Puebla. México.

***Médico Ultrasonografista. Hospital de la Madre y el Niño Indígena Guerrerense. Secretaría de Salud de Guerrero. Guerrero. México.

****Médico Anestesiólogo. Director Médico. Hospital Integral de Tecomatlán. Secretaria de Salud de Puebla. Puebla. México.

*****Médico de Urgencias. Hospital Integral de Tecomatlán. Secretaría de Salud de Puebla. Puebla. México.

Correspondencia: Dr. Indalecio Gustavo Martínez Velasco. Dirección: 27 Poniente No. 221. Col. El Carmen C.P. 72550. Puebla. México. Teléfono: +5212221168758. Correo electrónico: indaleciomvgine@outlook.com
\end{abstract}

\section{Resumen}

Los relámpagos se presentan con una frecuencia mayor a 50 veces por segundo a nivel mundial; sin embargo, las descargas directas suceden rara vez y son responsables del $5 \%$ de los eventos fatales. El mecanismo de lesión inicial es la parálisis de los centros vitales, lo que ocasiona apnea, fibrilación auricular o paro cardíaco. En mujeres embarazadas, el accidente por rayo puede producir complicaciones graves, dependiendo del punto de entrada en el cuerpo y su efecto indirecto sobre el feto, produciendo trastornos de la conducción en el sistema cardíaco y daño a nivel del sistema nervioso central. En la literatura mundial, solo catorce casos de embarazo y traumatismo por relámpago han sido reportados, sobreviviendo en todos la madre, con un único reporte de muerte fetal. Se presenta un caso de accidente por relámpago en una mujer embarazada en la semana 23 de gestación, cuyo tipo de lesión fue por contacto directo en el abdomen, y quien ingresó con compromiso neurológico, taquicardia materna y bradicardia fetal. El deceso materno ocurrió por infarto agudo extenso del miocardio y la formación de un émbolo intracavitario en el ventrículo izquierdo, con la consecuente muerte del binomio. Se describe su frecuencia, el mecanismo de lesión, las manifestaciones clínicas y el manejo actual. MÉD.UIS.2019;32(1):21-6

Palabras clave: Relámpago. Traumatismo por acción del relámpago. Muerte fetal. Muerte materna.

\section{Incidental maternal death due to lightning injury: a case report}

\begin{abstract}
Lightning occurs with a frequency rate of more than 50 times per second worldwide. Nevertheless, direct lightning strikes are not common, and they are responsible for about $5 \%$ of all lightning injury fatalities. The initial mechanism of injury is the paralysis of the vital centers, which causes apnea, atrial fibrillation and cardiac arrest. In pregnant women, lightning can produce severe complications which depend on the point of entrance and its indirect effect on the fetus, affecting the cardiac conduction system and the central nervous system. We report a case of a lightning accident by direct strike on a 23-week pregnant woman, who's type of injury was by direct contact on the abdomen. She arrives to the emergency room with neurologic symptoms, maternal tachycardia and fetal bradycardia. The mother died due to myocardial infarction and the presence of an intracavitary embolus in the left ventricle, with the consequent maternal-fetal death. The incidence and injury mechanisms, as well as the clinical features and clinical management are described. MÉD.UIS.2019;32(1):21-6
\end{abstract}

Keywords: Lightning. Lightning strike. Fetal death. Maternal death. 
¿Cómo citar este artículo?: Jiménez-López R, Martínez-Velazco IG, Rosas-Díaz JA, Veliz-Pradel N, Cruz-Rivera A. Muerte materna incidental en un accidente por relámpago: Reporte de caso. MÉD. UIS.2019;32(1):21-6. doi:10.18273/revmed.v32n1-2019003

\section{Introducción}

El relámpago o rayo es una descarga de corriente eléctrica como resultado de un desequilibrio entre la carga eléctrica de las nubes y la superficie terrestre'. Aproximadamente uno de cada cinco resulta en una descarga a la superficie terrestre, impactando de 0.2 a 0.8 personas por un millón de habitantes al año a nivel mundial, provocando 374 muertes entre 1995 y el $2000^{2}$. En América Latina, de acuerdo a los reportes emitidos por el Instituto de Investigación Nacional del Espacio de Brasil, este país tiene una de las más altas incidencias del planeta con 50 millones de relámpagos por año, provocando 75 muertes en solo $2008^{3}$.

Por su parte, de acuerdo con el Centro Nacional de la Prevención de Desastres, México es el país en el que más se registran decesos por este fenómeno natural en América Latina, con una cifra de 223 muertes anuales por relámpago, ocurriendo el $42 \%$ en el Estado de México.

Las complicaciones más peligrosas, y posiblemente letales, ocasionadas por este tipo de lesión son las cardiovasculares y las neurológicas ${ }^{5}$, provocando la muerte de aproximadamente una tercera parte de los $\operatorname{casos}^{6}$. De aquellos pacientes que sobreviven, $70 \%$ quedan con secuelas permanentes como parálisis, afasia, vértigo, alteraciones de la sensibilidad, e hipoacusia ${ }^{7,8}$.

La fisiopatología exacta del daño eléctrico en el humano no está bien aclarada, debido a que una gran cantidad de variables no pueden ser medidas o controladas cuando la energía eléctrica pasa a través del tejido. Con daños por altos voltajes, la mayoría de los estudios histológicos revelan coagulación y necrosis consistentes con daño térmico ${ }^{9}$. Por su parte, Lee et al. propusieron la teoría de la electroporación, en la cual las cargas eléctricas demasiado pequeñas producen daño térmico que causa cambios configuracionales de las proteínas, alterando la pared celular y su función. De esta forma, la naturaleza y la severidad de la lesión térmica eléctrica están en relación directa con los siguientes factores: tipo de circuito, duración, resistencia de los tejidos, voltaje, amperaje y trayectoria de la corriente?.

La posibilidad de presentación en la mujer gestante es un evento raro, documentándose en la literatura un total de catorce casos previamente reportados de accidentes por relámpago durante el embarazo; junto con este estudio sumarían un total de quince a nivel mundial ${ }^{10}$. En todos la madre sobrevivió, y solo hubo un caso de muerte fetal intrauterina.

Cuando el accidente de relámpago sucede en una embarazada, la posibilidad de morir la madre es mayor cuando la lesión ocurre por golpe directo del relámpago"1. En el caso del feto, la trayectoria vertical del relámpago en el cuerpo, es decir manopie o cabeza-pie, parecen llevar el mayor riesgo al producto, ya que el rayo atraviesa el útero, la placenta y el líquido amniótico, con la consecuente posibilidad de muerte fetal ${ }^{8}$.

A pesar de que un accidente por rayo es un evento raro, la repercusión en el binomio es de primordial importancia, por lo que el personal de salud de los diferentes niveles de atención debe conocer las principales lesiones que ocasiona, su evolución clínica y su abordaje y manejo en la mujer embarazada, para brindar un manejo oportuno con el propósito de salvaguardar el bienestar maternofetal'16-19. Teniendo esto en cuenta, se describe la evolución clínica de una paciente gestante con un accidente por relámpago, la cual falleció al presentar acinesia ventricular por un infarto extenso y la presencia de un coágulo intracavitario en el ventrículo izquierdo, con la consecuente muerte fetal.

\section{Presentación del caso}

Paciente de 23 años de edad residente del municipio rural de Cualac, Guerrero, México; con antecedente obstétrico $\mathrm{G}_{5} \mathrm{P} 4$, y embarazo de 23 semanas de gestación sin control prenatal; quien al realizar sus labores fuera de su domicilio, fue alcanzada por descarga eléctrica de relámpago, por lo que fue llevada inicialmente al centro de salud local, en donde al realizar la valoración inicial, encuentran 
compromiso en su estado neurológico con Glasgow 9/15 puntos, con confusión y letargia, midriasis, hipotermia, taquicardia materna y variabilidad en el registro de la frecuencia cardíaca fetal; por lo que fue referida al Hospital de la Madre y el Niño Guerrerense, ingresando 5 horas 40 minutos después de haber sufrido el evento. A su ingreso se activó Código Mater (Alarma obstétrica) y Equipo de Respuesta Obstétrica Inmediata ${ }^{12}$.

A la exploración física se hallaron los siguientes signos vitales maternos: TA 132/82 mmHg, FC 132 latidos/min, FR 44 respiraciones/min, SatO2 65\%, Temperatura $35.5^{\circ} \mathrm{C}$, con Glasgow de $12 / 15$ puntos (O: 4, V: 2, M: 6), combativa; llenado capilar de 3 segundos, con taquicardia, taquipnea, estertores en marea y esputo asalmonado. A nivel de abdomen superior se observan lesiones de Litchinger, lo que hace suponer que la descarga directa del relámpago sucedió a este nivel. Se encontró fondo uterino de 24 $\mathrm{cm}$, con frecuencia fetal de 100 latidos/min. La paciente requirió intubación orotraqueal y manejo ventilatorio avanzado, colocación de catéter central con restricción hídrica, manejo con dobutamina y, dados los hallazgos en el trazo electrocardiográfico con desniveles positivos del ST en la cara diafragmática (D II, D III y AVF), se instauró manejo convencional del infarto agudo de miocardio, y se realizó ecocardiograma Doppler, que reportó acinesia de la pared ventricular, secundaria a un infarto extenso, con la presencia de coágulo intracavitario extenso a nivel del ventrículo izquierdo de $52.8 \mathrm{cc}^{3}$ (Ver Figuras 1 y 2). De igual forma, la ultrasonografía fetal reportó un embarazo de 23 semanas de gestación con bradicardia fetal de 110 latidos/min.

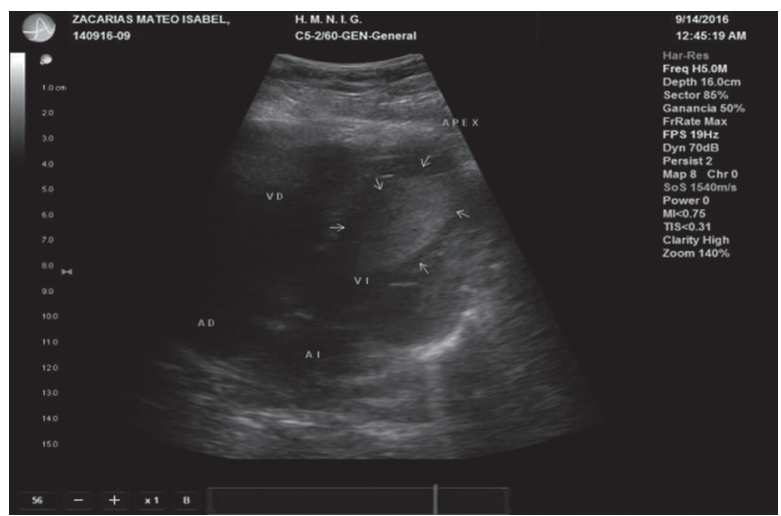

Figura 1. Coágulo intracavitario en ventriculo izquierdo en modo 2D en Ecocardiograma Doppler. Fuente: Autores.

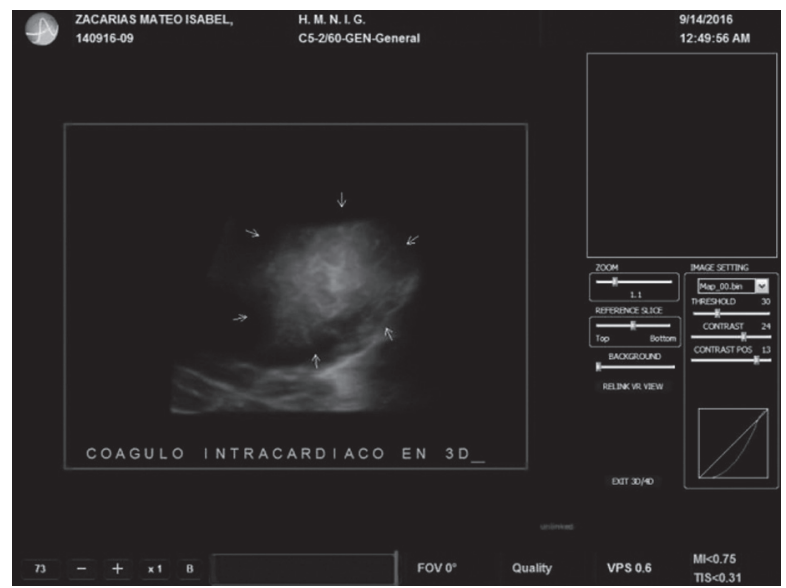

Figura 2. Coágulo intracavitario en ventrículo izquierdo en modo 3D en Ecocardiograma Doppler.

Fuente: Autores.

Finalmente la paciente presentó paro cardíaco, y fallece a pesar de la reanimación avanzada médicofarmacológica realizada de acuerdo a lo normado en el protocolo de atención ACLS, corroborándose al mismo tiempo muerte fetal con Doppler.

\section{Discusión}

Según Rees ${ }^{13}$, la primera descripción clínica de accidentes por relámpago se efectuó por Holes en 1830, quien menciona que los accidentes por relámpagos eran eventos raros, con una ocurrencia en aquella época de 44 casos por año en Inglaterra. Posteriormente, Dunscombe-Honiball en 1900, describió su experiencia durante 30 años de práctica clínica, analizando los casos y efectuando estudios post-morten en cada uno de ellos, logrando detallar magistralmente los hallazgos a nivel del corazón ${ }^{14}$. Con relación a la presentación durante la gestación, Schieffer es el autor que reporta el primer caso de una mujer embarazada afectada por un relámpago, en $1833^{15}$.

\section{Efectos maternos}

\section{Lesiones térmicas y quemaduras}

Desde el punto de vista clínico, las lesiones en piel por relámpago presentan cuatro patrones: lineal, en puntilleo, figuras de Lichtenberg y térmicas; pudiendo presentarse más de un patrón en cada víctima. Debido a la naturaleza superficial de muchas de estas quemaduras, pueden tomar varias horas 
para manifestarse clínicamente ${ }^{20,21}$. En este caso se documentó la presencia de las figuras de Lichtenberg, las cuales se presentaron inmediatamente en la zona abdominal, donde se localizó el punto de entrada del relámpago. Estas lesiones, también conocidas como floreadas, o en helecho, son patognomónicas del accidente por relámpago, siendo el tipo de lesión dérmica más frecuente. Son transitorias y se resuelven dentro de minutos a horas, tienen un patrón levemente palpable, de color café y rosa. No son realmente quemaduras ya que no dañan la piel, y parecen ser causadas por los electrones que dibujan un patrón debido al relámpago ${ }^{21}$.

La quemaduras reportadas de primer y segundo grado se presentan rara vez, documentándose en la literatura un caso de quemaduras de primer y segundo grado en Ghana ${ }^{21} \mathrm{y}$ uno de tercer grado por Zafren et al., las cuales ocurrieron principalmente en los orificios de entrada y salida ${ }^{22}$. Estos puntos de entrada y salida son raros en los accidentes por relámpago, sobre todo en los casos leves a moderados. En nuestra paciente solo se observó orifico de entrada, sin poder documentarse orificio de salida.

Se ha reportado que los pacientes con patrones de quemadura en la cabeza y la cara tienen un porcentaje mayor de mortalidad ${ }^{22}$. Sin embargo la mortalidad también puede presentarse cuando la descarga ocurre en el abdomen, como sucedió en este caso.

\section{Sistema cardiovascular}

La causa principal de muerte en el accidente por relámpago es el paro cardiorrespiratorio, siendo demostrado que la fibra miocárdica es despolarizada cuando el relámpago golpea, y que el corazón permanece contraído durante el tránsito de la energía en el tejido cardíaco. El paro cardíaco es generalmente asistólico, como fue el caso en nuestro reporte, y puede ser temporal si se da una reanimación cardiopulmonar adecuada. El paro cardíaco secundario ocurre por una parada en el centro respiratorio, conduciendo a asfixia y al desarrollo de arritmias ventriculares ${ }^{23-27}$. Si el paciente no fallece por esta causa, es raro que las víctimas mueran por otra condición en la etapa aguda.

La lesión cardíaca después del accidente por relámpago puede manifestarse con inversión en las ondas $T$ en el electrocardiograma, que se observan en la cara anterior e inferior, siendo un hallazgo sugestivo de isquemia, siendo más por inestabilidad autónoma que por la lesión miocárdica propiamente dicha ${ }^{23-27}$. En la paciente de nuestro reporte se presentaron ondas $T$ invertidas en las derivaciones V 5 y V6, observándose así una zona isquémica en la cara anterolateral izquierda.

\section{Sistema nervioso}

La amnesia anterógrada y la confusión ocurren comúnmente entre las víctimas de accidentes por relámpago, quedando incapaces de recordar hechos por minutos o días ${ }^{28-31}$. La paciente a su ingreso presento confusión y letargia, las cuales continuaron hasta su deceso. Las parestesias transitorias, disestesias y espasmos musculares, son también comunes, pues las afecciones del sistema nervioso periférico se localizan principalmente a nivel de las extremidades, reportándose incluso algunos casos de plexopatía braquial32. En algunos pacientes se puede presentar cefalea postraumática, la cual generalmente se resuelve dentro de unos pocos meses. En nuestra paciente el deceso inmediato impidió observar estas complicaciones.

Entre las condiciones crónicas que se pueden presentar después de un accidente por rayo se encuentran: neuropatía periférica, disfunción motora de habilidades finas, síndrome de dolor crónico, trastornos del sueño y síntomas neuropsicológicos, incluida la depresión y el estrés postraumático ${ }^{29}$. Adiconalmente, la trayectoria de la corriente eléctrica a través del cerebro puede producir hematoma epidural, subdural o hemorragia intraventricular, e incluso se ha reportado un caso de infarto cerebelar en la literatura ${ }^{33}$. Sin embargo, debido al el deceso súbito de la paciente, solo se reconocieron alteraciones agudas en este sistema.

Por otra parte, una contusión postraumática, sea por caídas o lesión por onda de choque, puede causar lesiones ortopédicas y hemorragias internas leves, por lo que se debe investigar diligentemente en estas pacientes la posibilidad de fracturas o choque hemorrágico. Además, el daño por onda de choque comúnmente causa sordera transitoria, donde casi $50 \%$ de las víctimas presentan ruptura de uno o ambas membranas timpánicas ${ }^{31}$.

\section{Efectos fetales}

Las principales alteraciones encontradas en los fetos en el reporte de Galster et al., fueron las alteraciones del ritmo fetal, principalmente bradicardia ${ }^{10}$, como se 
diagnosticó en el feto de nuestra paciente. De igual forma, teniendo en cuenta que la piel del feto es menos resistente al pasaje de la corriente eléctrica que la piel materna por estar humedecida, ésta ofrece 200 veces menos resistencia al paso de la corriente eléctrica que la piel postnatal, y el riesgo de daño fetal a su sistema de conducción cardíaca es mayor ${ }^{10}$. La muerte fetal en el caso actual fue probablemente debida a una interrupción súbita de la circulación materna asociada a la inmadurez extrema.

\section{Abordaje actual}

La reanimación inmediata es el factor más importante en el manejo en este tipo de accidentes. En la escena de un accidente por relámpago, la atención médica debería ser dirigida hacia la víctima, aunque aparentemente esté muerta. En nuestro reporte, el retraso en el traslado de la paciente al hospital ocasionó el deceso de ambos, a pesar del abordaje especializado ${ }^{12}$. De esta forma, se corrobora que el tiempo de traslado es el factor de riesgo más importante para la sobrevida materna y fetal en estos casos, como ha sido reportado por Cooper, et $a^{30}$.

La atención médica dependerá del lugar de ocurrencia, siendo una prioridad instalar el soporte vital avanzado brindándose inicialmente la valoración del estado neurológico, estado hemodinámico-ventilatorio y terapia personalizada. Las medidas de reanimación cardiopulmonar y el soporte básico y avanzado deben ser establecidos tan pronto como sea posible después del accidente, manteniendo apoyo cardiocirculatorio, la vía aérea permeable y ventilación con aporte suplementario de oxígeno adecuado. La reanimación cardiopulmonar debería ser realizada en un periodo mayor de tiempo, si es necesario, ya que los pacientes con paro cardiopulmonar secundario a accidente por relámpago tienen mejor pronóstico que aquellos con paro cardiopulmonar secundario a otro tipo de lesión ${ }^{34}$.

\section{Valoración del bienestar fetal}

Es importante la valoración exhaustiva del bienestar fetal, sobre todo si el accidente sucedió en el tercer trimestre, ya que en este período es mayor la posibilidad de muerte intrauterina, ruptura uterina o inducción de labor. La paciente de este reporte cursaba el segundo trimestre de gestación, con un feto inmaduro, siendo más susceptible a los efectos de la intensidad de la corriente, lo que ocasionó su deceso. Además, la valoración neurológica del recién nacido debe incluir tomografía y/o resonancia magnética por la posibilidad de isquemia en el sistema nervioso central.

De igual forma, el ultrasonido servirá para valorar la edad gestacional y el perfil biofísico. La resolución del nacimiento dependerá de las condiciones de la madre y del feto, siendo la sobrevida fetal en los casos reportados de buen pronóstico.

\section{Conclusiones}

En caso de presentarse lesión por accidente de relámpago en la mujer embarazada se sugiere un manejo inmediato a la madre desde el lugar del accidente con soporte vital básico y avanzado, realizando el traslado oportuno a una unidad hospitalaria con mayor poder de resolución del caso, sin importar el nivel de atención del primer contacto. El bienestar fetal debería ser prontamente evaluado después del accidente sin considerar que tan trivial pareciera ser el evento. La vía y momento del nacimiento dependerá del estado de bienestar fetal y su edad gestacional. Por otro lado, es una prioridad contar con un programa nacional de prevención de este tipo de accidentes naturales, para educar a la población general y evitar muertes prevenibles.

\section{Consideraciones éticas}

El autor principal ha obtenido el consentimiento informado de los familiares de la paciente.

\section{Declaración de autoría}

Todos los autores han contribuido intelectualmente al trabajo, habiendo investigado la bibliografía, la elaboración de las partes del artículo y la evaluación final.

\section{Referencias bibliográficas}

1. Boren D. National Oceanic and Atmospheric Administration. NSSL [internet]. 2002 [citado 2018]. Disponible en: http:// www. Nssl.noaa.gov/primer/lightning/ltg_climatology.html

2. Holle R. Annual rates of lightning fatalities by country: International Lighting Detection Conference. Visalia [internet]. 2008 [citado el 20 jun 2016]. Disponible en: http://www.vaisala. com/Vaisala\%20Documents/Scienti[c\%20papers/Annual_rates_ of_lightning_fatalities_by_country.

3. Forster SA, Silva IM, Ramos MLC, Gragnani A, Ferreira LM. Lightning burn: Review and case report. Burns. 2013;39 (2):8-12.

4. Muertes por rayo en Mexico. Cenaced [internet] 2017 [citado el 16 jul 2017]. Disponible en: http://www.cenaced.org.mx/es/blog/ item/90-mueren-mas-por-rayos-en-mexico-que-en-otras-partesdel-mundo.html 
5. Pierce MR, Henderson RA, Mitchell JM. Cardiopulmonary arrest secondary to lightning injury in a pregnant woman. Ann Emerg Med. 1986; 15(5):597-99.

6. Chan YF, Sivasamboo R. Lightning accidents in pregnancy. J Obstet Gynaecol Br Commonw. 1972; 79(8):761-62.

7. Guha-Ray DK. Fetal death at term due to lightning. Am J Obstet Gynecol. 1979;134(1):103-5.

8. Browne BJ, Gasch WR. Electrical injuries and lightning. Emerg Med Clin North Am. 1992;10(2):211-29.

9. Andrews CJ, Cooper MA, Darveniza M, Mackerras D. Lightning Injuries: Electrical, Medical and Legal Aspects. Boca Raton: CRC Press; 1992.

10. Galster K, Hodnick R, Berkeley RP. Lightning strike in pregnancy with fetal injury. Wilderness Environ Med. 2016; 27(2): 287-90.

11. Ritenour AE, Morton MJ, McManus JG, Barillo DJ, Cancio LC. Lightning injury: a review. Burns 2008— 4(5):585-94.

12. Secretaria de Salud, Centro Nacional de Equidad de Género y Salud Reproductiva. Triage Obstétrico, Código Mater y Equipo de Respuesta Inmediata Obstétrica. Lineamiento Técnico 2016. Ciudad de México: Delegación Miguel Hidalgo; 2016.

13. Rees WD. Pregnant woman struck by lightning. Br Med J. 1965; 1(5427): 103-4.

14. Dunscombe-Honiball O. Accidents and injuries caused by lightning. Br Med J. 1900; 1(2054): 1153-5.

15. Schieffer (1833). Quoted by Stricker, Virchow's Arch Path Anat. 1861(20):54.

16. Navarro Escayola E. Valoración médico legal de lesiones y muerte por fulguración. Gac Int Cienc Forense. 2012; 5:8-18

17. Zack F, Rotthschid MA, Wegener R. Lightning Strike-Mechanisms of Energy Transfer, Cause of Death, Types of Injury. Dtsch Arztebl 2007; 104(51-52):A 3545-9.

18. Pfortmueller CA, Yikun Y, Haberkern M, Wuest E, Zimmermann $\mathrm{H}$, Exadaktylos AK. Injuries, sequelae, and treatment of lightninginduced injuries: 10 years of experience at a swiss trauma center. Emerg Med Int. 2012;2012: 167698.

19. Meza-Rojas FG, Rodríguez-Cázares LC. Lesiones por fulguración: aspectos clínicos y médicos legales. Rev Fac Med UNAM. 2017; 60 (2):11-6.

20. Murty OP. Lightning fatality with blast, flame, heat and current effects: a macroscopic and microscopic view. J Forensic Leg Med. 2009; 16(3): 162-7.

21. Apanga PA, Azunah JA, Yiranbon JB. A rare manifestation of burns after lightning strike in rural Ghana: a case report. J Med
Case Rep. 2017; 11(1):200-2.

22. Zafren K, Durrer B, Herry JP, Brugger H. Lightning injuries: prevention and on-site treatment in mountains and remote areas: official guidelines of the International Commission for Mountain Emergency Medicine and the Medical Commission of the International Mountaineering and Climbing Federation (ICAR and UIAA MEDCOM). Resuscitation. 2005; 65(3):369-72.

23. Ruiz-Ruiz FJ, Ruiz-Laiglesia FJ, Lobo Escolar A, Hualde-Enguita AM, et al. Alteraciones cardiacas en la fulguración por impacto indirecto. Rev Esp Cardiol. 2002;55(7):768-70.

24. Arguelles-Arguello A, Barrantes Rodríguez K, González-Cerdas JP, León S, Umaña A, Vargas C. Fisiopatología, manifestaciones sistémicas y secuelas de la fulguración en seres humanos. Med. leg. Costa Rica. 2015; 32(1): 138-45

25. O'Keefe-Gatewood M, Zane RD. Lightning injuries. Emerg Med Clin North Am. 2004; 22(2): 369-403.

26. Ozgur M, Gunal K, Duzgunoglu-Boz S, Buyukkaya S. Myocardial and neurocognitive changes in lightning strike injury: a case report. Med. Res. Chron. 2014; 1(2): 169-72.

27. Cannell H. Struck by lightning: the effects upon the men and the ships of HM Navy. J R Nav Med Serv. 1979; 65(3): 165-70.

28. Waters D, Brotons C, Chiang C, Ferrières J, Foody J, Jukema J, et al. A Multinational Survey to Evaluate the Proportion of Patients Achieving Low-Density Lipoprotein Cholesterol Goals. Circulation. 2009;120(1);28-34.

29. Copper MA, Andrews CJ. Neuropsychological aspects of lightning injury. In: Proceedings of the 9th International Conference on Atmospheric Physics, St. Petersburg, Russia, June 1992.

30. Cooper MA. Emergent care of lightning and electrical injuries. Semin Neurol. 1995;15(3):268-78.

31. Turan M, Kalkan F, Bozan N, Özçalimli İ, Zeki M, Yalınkılıç A, et al. Isolated Sensorineural Hearing Loss as a Sequela after Lightning Strike. Case Rep Otolaryngol. 2015;2015: 738416

32. Bhargava A, Kasundra G, Khichar S, Bhushan B. Lightning strike-induced brachial plexopathy. J Neurosci Rural Pract. 2014; 5(4):399-400.

33. Aslan S, Yilmaz S, Karcioglu O. Ligthning: an unusual cause of cerebellar infarction. Emerg Med J. 2004; 21(6):750-1.

34. Biswas A, Dalal K, Hossain J, Baset KUJ, Raman F, Mashreky SR. Lightning Injury is a disaster in Bangladesh? - Exploring its magnitude and public health needs. F1000Res. 2016:5:2931-8. Published online 2016 Dec 29. Doi: 10.12688/ f1000research.9537. 